\section{Regulating STING}

The intracellular membrane-tethered protein STING couples sensors of the innate immune system to the production of type $I$ interferon by facilitating activation of the transcription factor IRF3 by the kinase TBK1. In Cell, Konno et al. identify a feedback regulatory mechanism that prevents sustained activation of STING and inflammatory disease. STING is activated by cyclic guanosine or adenosine dinucleotides (cGAMP) that are generated by cGAMP synthase (cGAS) after aberrant nucleic acids are detected. Cyclic dinucleotides likewise bind to the kinase AMPK, which acts as a suppressor of the autophagy protein ULK1 (ATG1). cGAMP causes AMPK to release tethered ULK1 that then phosphorylates STING, specifically on Ser366, and induces its degradation in lysosomes. ULK1-mediated targeting of STING thus prevents further activation of IRF3 and terminates the expression of type I interferon.

$L A D$ Cell 155, 688-698 (2013)

\section{Slipping the NET}

The important human pathogen Staphylococcus aureus displays a dazzling array of strategies for evading the immune system. In Science, Schneewind and colleagues demonstrate that $S$. aureus is also able to subvert neutrophil extracellular traps (NETs) to kill host macrophages. Neutrophils release NETs (tangles of nucleic acid interspersed with antimicrobial peptides) in response to bacteria, and this represents an important protective mechanism. Also important are macrophages, which phagocytose bacteria; however, the characteristic abcess of $S$. aureus almost completely lacks macrophages. S. aureus expresses two virulence factors, a nuclease and an adenosine synthase, which process NET DNA into cytotoxic 2'-deoxyadenosine. In the absence of antimicrobial peptides, $2^{\prime}$-deoxyadenosine alone is sufficient to trigger an intrinsic apoptosis pathway in macrophages but not in neutrophils. $S$. aureus is thus able to redirect an otherwise functional host antibacterial mechanism to destroy protective macrophages.

Science 342, 863-866 (2013)

\section{A window of allergy}

The microbiota of the intestine shapes both local and systemic immune compartments. In Cell Host and Microbe, McCoy and colleagues reveal that bacterial colonization of the neonatal intestine also influences long-term production of immunoglobulin E (IgE). Germ-free mice develop progressively increasing serum IgE titers, whereas other immunoglobulin isotypes are unaffected. That increase in IgE is dependent on the presence of both CD4 ${ }^{+}$ T cells and interleukin 4 (IL-4) but cannot be normalized by adoptive transfer of regulatory $T$ cells ( $T_{\text {reg }}$ cells) or treatment with IL-10. Furthermore, germ-free mice fed an antigen-free diet also develop 'hyper-IgE', which rules out the possibility of the presence of an oral allergen. Instead, the dysregulated IgE production seems to result from diminished diversity of intestinal microbes. Accordingly, exposure of germ-free mice to a diversified microbiota during a short neonatal window is sufficient to forestall switching to IgE. Therefore, diversity of the intestinal microbiota early in life also seems to set the propensity to spontaneously develop IgE and, potentially, systemic allergy. ZF

\title{
Unstable Foxp3
}

Loss of the transcription factor Foxp3 in $\mathrm{T}_{\text {reg }}$ cells has been noted in various inflammatory conditions. In Immunity, Bluestone and colleagues use an experimental autoimmune encephalitis (EAE) model to show that antigen-driven activation and inflammation selectively promotes Foxp3 instability in autoreactive $\mathrm{T}_{\text {reg }}$ cells. Antigen-driven activation in an inflammatory environment results in downregulation of Foxp3 transcription in $\mathrm{T}_{\text {reg }}$ cells that had high Foxp3 expression before EAE induction. Formerly $\mathrm{T}_{\text {reg }}$ cells that have lost Foxp3 expression produce interferon- $\gamma$ in amounts similar to those observed in pathogenic effector T cells and induce EAE after adoptive transfer. Supplementation with IL-2 stabilizes Foxp3 expression in antigen-specific $T_{\text {reg }}$ cells and protects mice from disease. Thus, the stability of antigen-specific $T_{\text {reg }}$ cells might be important for the course and control of autoimmunity.

Immunity 39, 949-962 (2013)

\section{Autoreactive T cell trafficking}

The immunomodulatory receptor CTLA-4 maintains tolerance to self, and $\mathrm{Ctla}^{-/-}$mice develop lymphoproliferative disorders with tissue infiltration of self-reactive T cells. In Nature Medicine, Kang and colleagues show that signaling via the coreceptor CD28 and the signaling kinase Itk specifically regulates the migration of self-reactive $\mathrm{T}$ cell to tissues. The authors show that interactions of CD28 with its ligand B7, between $\mathrm{T}$ cells and $\mathrm{B} 7$-expressing endothelial cells, modulate the trafficking of $\mathrm{Ctla4}^{-/-} \mathrm{T}$ cells. Downstream of CD28, Itk is required for the transendothelial migration of $\mathrm{Ctla}^{-/-} \mathrm{T}$ cells, although it is not required for the activation of $\mathrm{Ctla4}^{-/-} \mathrm{T}$ cells or for exit from the lymph nodes. In addition, Itk does not modulate the activity of $\mathrm{Ctla4}^{-/-} \mathrm{T}_{\text {reg }}$ cells. An inhibitor of Itk diminishes the autoimmune tissue infiltration in $\mathrm{Ctla}^{-/-}$mice and the infiltration and destruction of beta islets in mice of the nonobese diabetic strain, which results in a lower incidence of diabetes. 